Gut, 1963, 4, 273

\title{
The clinical features of haemorrhage from diverticula of the colon
}

\author{
M. W. F. DUNNING ${ }^{1}$ \\ From the Central Middlesex Hospital, London, Broadgreen Hospital, \\ Liverpool, and the Royal Southern Hospital, Liverpool
}

EDITORIAL SYNOPSIS Haemorrhage is an occasional but sometimes serious complication of diverticulitis. This paper is a clinical study and discusses surgical treatment. Defunctioning colostomy is advocated when possible as an alternative to emergency resection.

The first recorded case of haemorrhage from diverticulitis was reported by Koch (1903). A man aged 64 gave a history of abdominal pain, constipation, and considerable rectal bleeding for one year. Examination revealed a tender mass in the left iliac fossa. Carcinoma of the sigmoid colon was considered the obvious diagnosis and colostomy was performed. The patient died from peritonitis and a post-mortem examination of the mass revealed diverticulitis with no evidence of carcinoma. This was a most unusual record as it was the practice at this period to regard such lesions as obvious carcinoma without further examination.

Despite this instructive and early report, little attention has been directed to haemorrhage from diverticula of the colon. Maxwell Telling (1908) made no reference to haemorrhage in his paper on the pathological changes and clinical results of acquired diverticula of the sigmoid colon, but in a later review of 324 published cases Telling and Grüner (1917) stated: 'Though blood has been noted in some cases, yet its presence is so exceptional that it should give rise to the greatest reluctance in diagnosing diverticulitis as opposed to carcinoma. Its absence is of course due to the fact that the mucosa of the bowel is so seldom involved.' This mistaken conclusion was clearly due to the fact that at this period the majority of cases of diverticulitis were wrongly diagnosed as carcinoma. Nevertheless bleeding continued to be regarded as excessively rare in diverticular disease and it is only during recent years that there has been some recognition of the fact that haemorrhage is by no means a rare complication.

In 1944 Young and Young reported bleeding in $26 \%$ of a series of 91 cases of diverticular disease. Two years later Morton (1946) reported haemorrhage

\footnotetext{
1 Now at St. Mark's Hospital, London.
}

as a presenting or major symptom in $21.6 \%$ of 111 cases; Bacon and Sherman (1950) noted an incidence of $18 \%$ in 117 cases.

Noer (1955) assessed the incidence of macroscopic rectal bleeding in 2,896 recorded cases of diverticulosis and diverticulitis and found it to be at least $11 \%$, but in his own series of 68 cases $29 \%$ had bleeding as a presenting or major symptom. In a similar survey Rushford (1956) found that haemorrhage occurred in $17 \%$ of 6,000 recorded cases of diverticulitis and in a personal series of 120 cases bleeding had been a symptom in $27.5 \%$ of the cases. Keith and Rini (1957) reported haemorrhage in $19 \%$ of 317 cases of diverticular disease, and Mobley, Dockerty, and Waugh (1957) found that the incidence of haemorrhage in 1,970 cases from the Mayo Clinic was $7 \cdot 9 \%$.

\section{MATERIAL}

The 249 patients in this series include 91 cases examined personally during the seven-year period 1954-61 and 158 unselected cases studied from their records (1950-60). All of the patients were in-patients with symptoms attributed to diverticular disease and all were fully investigated.

Six of the patients have been followed up for two years only, but all the remaining cases have been followed up for at least three years without reason to change or modify the diagnosis.

\section{INCIDENCE OF HAEMORRHAGE}

Macroscopic rectal bleeding occurred in 35 of the 249 patients studied, an incidence of $14 \%$. The haemorrhage was severe enough to demand transfusion in nine cases. In a further three cases transfusion was indicated, as the haemoglobin level 
dropped to below $55 \%$, though the anaemia was in fact corrected with oral and parenteral iron.

Haemorrhage in diverticular disease of the colon may be mild, moderately severe, or exsanguinating.

MILD HAEMORRHAGE The majority of haemorrhages from diverticula of the colon are included in this category, in which unequivocal macroscopic blood, recognizable as such by the patient, is passed per rectum. The blood loss is usually unimportant and the bleeding generally ceases spontaneously, but there may be a persistent discharge of small quantities of blood for a considerable period. The bleeding may recur, either with or without other bowel symptoms, and may be related or unrelated to defaecation. In this type of bleeding the diagnosis can be made only by excluding other causes, particularly polypi and carcinoma. Haemorrhoids are of course easily excluded.

Patients with diverticulosis in which small quantities of altered blood have been discovered only by sigmoidoscopy on the unprepared bowel may have an associated neoplasm. Although diverticulitis alone may be the cause, such a finding is most suspicious and Lloyd-Davies (1953) found new growth associated with an area of diverticulitis in six out of nine such cases. Four of the six new growths were malignant. In the remaining three cases no tumour was found, but severe diverticulitis was present. This type of bleeding has not been included as bleeding from diverticula of the colon in this investigation; neither have cases in which occult blood only was found.

Case 1 Mrs. E. M., aged 73, had been very well and symptom free until 24 February 1959 when she was awakened during the night by a sudden desire to have her bowels moved; she then had a small rectal haemorrhage of bright red blood. She passed blood clot on the following day, but has passed no blood since. There was no pain or diarrhoea. Examination and sigmoidoscopy revealed no abnormality. Barium enema showed diverticulosis of the pelvic colon. No other abnormality was found. She has had no further symptoms and remains well.

Case 2 Miss H. W., aged 65, complained on 1 January 1958 of passing bright red blood per rectum intermittently for two weeks. There had recently been a tendency to constipation, otherwise she had been well. Physical examination revealed no abnormality. Sigmoidoscopy demonstrated diverticula with a small associated granuloma at $19 \mathrm{~cm}$. The 'tumour' was removed and histology confirmed that it was a mass of granulation tissue. A barum enema showed diverticulitis of the sigmoid flexure, but no other abnormality. She has remained symptom free.

Case 3 Mrs. L. H., aged 62, had experienced three episodes of discomfort in the left iliac fossa followed by passage of bright red blood per rectum over the past 10 years. The bleeding always ceased spontaneously after a few days. Repeated investigations had only shown diverticulosis of the sigmoid colon. On 29 December 1960 she had a further rectal bleed. Sigmoidoscopy showed no abnormality. The latest barium enema (3 January 1961) showed diverticulosis only, though the number of diverticula have increased over the past 10 years.

MODERATELY SEVERE HAEMORRHAGE The clinical picture in this group is much more specific and the haemorrhage is less likely to be confused with bleeding from carcinoma of the colon. The haemorrhage usually starts with dramatic suddenness. The patient experiences an unexpected call to stool and passes bright red blood in considerable quantity per rectum.

There are two distinct forms of this type of bleeding: 1 A sudden severe rectal haemorrhage which stops as dramatically as it started, with no further bleeding; 2 a series of episodes of bleeding, sometimes associated with diarrhoea. The blood loss often amounts to several pints and the subjective symptoms depend upon the amount and rate of loss. The sudden loss of one or more pints of blood causes some degree of shock. In group 2 each episode of bleeding is usually less severe than the single profuse haemorrhage of group 1. The blood loss in these cases is less sudden, but the cumulative volume over a longer period may be greater. These patients usually present without shock, but with obvious anaemia; they may, however, present with classical shock following the sudden loss of a considerable quantity of blood after earlier less severe bleeds.

Case 4 Mrs. F. H., aged 63, was admitted to hospital on 26 August 1956 following a severe rectal haemorrhage. She had been perfectly well until this event and there were no other symptoms. She was very pale; the haemoglobin level was $48 \%$. She was transfused with 3 pints of blood. Six hours after admission she had a further rectal haemorrhage and laparotomy was performed. The only abnormality found was extensive diverticulosis involving the whole large bowel. The abdomen was closed without further surgery ; 2 more pints of blood were transfused and conservative treatment was continued. The bleeding ceased spontaneously. She has had no further bleeding and remains well and symptom free. Investigation and follow-up (four years) have only shown extensive diverticulosis of the colon. There is no evidence of superimposed diverticulitis.

Case 5 Mr. G. D., aged 64, was admitted to hospital on 16 July 1959 following four episodes of profuse rectal haemorrhage of bright red blood which had occurred at intervals during the preceding month. On each occasion the bleeding was profuse and accompanied by breathlessness. He had been very well and entirely symptom free before the first haemorrhage. On admission he was complaining of breathlessness with anginal pain on exertion. He was very pale; his haemoglobin level was $37 \%$. Red blood cells showed marked hypochromia and microcytosis. White blood cells and platelets were 
normal. The E.C.G. was normal. Clinical examination and sigmoidoscopy revealed no abnormality. He was transfused with 5 pints of blood. Subsequent investigations and barium studies only revealed diverticulosis of the pelvic colon. He has had no further bleeding and has remained well and entirely symptom free.

EXSANGUINATING HAEMORRHAGE Haemorrhage from diverticulitis, and apparently from diverticulosis, may present in a massive exsanguinating form. Death has resulted from such haemorrhage. The largest haemorrhage from diverticulitis that was encountered (reported below) necessitated the transfusion of 30 pints of blood (including 6 pints of packed cells) and surgery was eventually necessary to arrest the haemorrhage. Massive haemorrhage may occur from diverticula of the colon without any other symptoms. This usually occurs in a middle-aged or elderly person who appears to be quite well, and the bleeding often, but by no means always, ceases spontaneously. Full investigations only reveal diverticula of the colon. There may not be obvious diverticulitis. This presentation was first described by Germain, Le Gallou, and Morvan (1938). Fraenkel (1954) found 15 cases of diverticulosis presenting in such a manner in a survey of 366 relevant case histories of patients admitted to the Radcliffe Infirmary, Oxford, during the five-year period 1946-51.

Case 6 Mr. A. G., aged 67, was admitted to hospital following a profuse rectal haemorrhage on 28 July 1954. He was very obese, but had been relatively fit. Five hours before admission he had an unexpected call to stool, and passed bright red blood per rectum in considerable quantity. This was followed by 10 similar incidents in the course of the next four hours. There was no pain or diarrhoea. He gave a history of passing occasional small quantities of blood per rectum over the preceding 10 years, but this had been attributed to haemorrhoids. He had experienced a tendency to constipation for the past six months.

On admission his pulse rate was 140 , and haemoglobin concentration 56\%. Proctoscopy revealed first degree haemorrhoids and that the source of bleeding was above the rectum. Sigmoidoscopy was uninformative owing to continued bleeding. He was at once treated with blood transfusion.

The bleeding continued and during five days he had received 15 pints of blood which included 6 pints of packed cells. Laparotomy was performed. The whole colon was distended with blood; there was no blood in the bowel proximal to the ileo-caecal valve. There was extensive diverticulosis involving the whole large bowel, with superimposed diverticulitis in the distal colon. There was no evidence of growth and it was not possible to locate the site of bleeding. The abdomen was closed and conservative treatment continued.

The bleeding still continued and after a further five days the patient's condition was causing anxiety. He had then been transfused with 27 pints of blood.

On the assumption that the bleeding was probably from the distal colon, where there was superimposed diverticulitis, a right transverse colostomy was performed and then the bleeding ceased. He had been transfused with 30 pints of blood including 6 pints of packed cells. The colostomy acted on the third post-operative day (12 August 1954) and at the same time a large mass of blood clot was passed per rectum. The colostomy continued to act well and by the sixth post-operative day, there was no macroscopic blood in the faeces from the colostomy.

Owing to the extensive nature of the lesion and the patients' obesity, excision was not advised.

He has remained in fairly good health ever since, although there has been recurrent bleeding (via the colostomy) on several occasions. These have been minor incidents with the exception of one, when he was admitted to hospital and transfused with 2 pints of blood.

A most careful follow-up of over six years with repeated sigmoidoscopies and barium enemata has given no new findings.

This patient demonstrates the severity of the bleeding that may occur from diverticula of the colon, that the haemorrhage may not cease spontaneously with conservative treatment, and a satisfactory result from a defunctioning colostomy.

Case 7 Mr. T. W, aged 65, was admitted to hospital on 23 April 1951 following a profuse rectal haemorrhage. He had complained of constipation for the past six months, but had otherwise been symptom free. On admission he was still bleeding per rectum. Sigmoidoscopy showed no local abnormality to $20 \mathrm{~cm}$. and that the bleeding was from a higher level.

He was treated conservatively and with blood transfusion. The bleeding continued and on 25 April the blood pressure dropped to $70 / 40 \mathrm{~mm}$. $\mathrm{Hg}$. On 30 April there was some anxiety about his condition and laparotomy was performed. The whole large bowel was distended with blood. There was a localized area of diverticulitis in the sigmoid colon and one diverticulum was acutely inflamed. The sigmoid colon was mobilized and exteriorized with resection of the area of diverticulitis thereby forming a colostomy.

Following this operation there was no further bleeding. The patient had been transfused with 10 pints of blood and 4 pints of plasma. Histological examination of the resected specimen showed diverticulitis only. The mucosa of the acutely inflamed diverticulum had sloughed in places as a result of the inflammatory changes. The colostomy was closed two months later and the patient has had no further trouble.

If laparotomy for continued bleeding reveals an obviously localized segment of diverticulitis, it is justifiable to resect it in the hope that the source of bleeding will thus be removed. This procedure has not always, however, been effective in controlling the bleeding under these circumstances. 
In the following case it was reasonably certain that the localized area of diverticulitis bearing an acutely inflamed diverticulum was the cause of the bleeding and the results of resection were excellent. Total colectomy, however, carries a considerable risk.

Case 8 Mr. H. C., aged 59, had had two episodes of rectal bleeding two years previously and was admitted on 5 January 1960 with a massive haemorrhage via the rectum, but no other symptoms. He was treated with blood transfusion.

The bleeding continued and during the next three days he received 14 pints of blood, but this failed to raise the level of his haemoglobin, and his condition was causing anxiety. Laparotomy was therefore performed on 8 January. The whole colon was the seat of extensive diverticulosis, and was distended with blood. There was no blood in the bowel proximal to the ileo-caecal valve. It was not possible to locate the site of bleeding and it was felt that an emergency colectomy would give the patient his best chance of survival.

Initially the patient's progress was satisfactory but then his general condition deteriorated and he died with bronchopneumonia a week after the operation.

A complete examination of the large bowel revealed diverticulosis with some superimposed diverticulitis. No other abnormality was found.

It is clearly wise to avoid extensive reflections during active bleeding if it is possible. There is no doubt that if a massive haemorrhage from diverticula of the colon fails to respond to conservative treatment the condition becomes grave, unless laparotomy reveals the site of the haemorrhage or that nothing more than a defunctioning colostomy is required. This case also shows that haemorrhage from diverticula of the colon may recur in a massive form after earlier less severe bleeds. If excisional surgery is feasible it should therefore be considered in the quiescent period following active bleeding.

\section{MECHANISM OF BLEEDING FROM DIVERTICULA OF THE COLON}

Noer (1955) has shown that there is a striking concentration of blood vessels in the vicinity of colonic diverticula. The illustrations of specimens of human colon (Fig. 1) prepared by clearing after injection of arteries and veins with red and blue liquid latex show very clearly the large blood vessels within the thin walls of the diverticula; their size and distribution is such as to make severe haemorrhage probable in the event of erosion or ulceration. If diverticulitis is present, bleeding is probably due to necrosis of a vessel wall following involvement in the inflammatory process. The degree of haemorrhage presumably varies with the size of the vessel involved.

Granulation tissue and congested mucosa may be the source of minor degrees of bleeding. I have on two occasions seen a vascular mass of granulation tissue through the sigmoidoscope in cases of bleeding from diverticulitis, but I was not convinced that this tissue was the source of the bleeding in either case.

In view of the absence of pyrexia, a raised white cell count, and other evidence of diverticulitis in most cases of haemorrhage from diverticula of the colon, there is some difficulty in deciding whether the underlying lesion is diverticulitis or simple diverticulosis. A review of all the relevant literature suggests that haemorrhage does sometimes occur in simple diverticulosis. In this respect it is interesting to note that massive haemorrhage may also occur from jejunal diverticulosis (Klidjian, 1946; Kozoll, McMahon, and Kiely, 1950; Waterson, 1952), in which condition inflammatory changes do not occur owing to the fluid nature and relative sterility of the proximal small bowel contents.

Local trauma to a vessel could theoretically cause haemorrhage in diverticulosis. Young and Beckett Howorth (1954) have reported a case of massive haemorrhage from diverticulosis of the colon which was treated by colectomy with a fatal outcome. Examination of the specimen showed that some of the diverticula were inverted and the mucosa of one of the inverted diverticula was ulcerated. An inverted diverticulum may more easily sustain local trauma and it has been suggested that inversion may be an important factor in these cases. However, Noer's demonstrations clearly show that the occurrence of bleeding is favoured by the size and distribution of the vessels alone and it is probable that inversion of diverticula is not an important factor in the cause of bleeding.

My own impression is that there is nearly always some superimposed diverticulitis in cases of bleeding from diverticula of the colon but there may be only a very small focus. In case 7 only one of the diverticula present was acutely inflamed, but the inflammatory reaction was severe enough to cause some sloughing of the mucosa. A similar acute inflammatory reaction involving a very small area of mucosa at the mouth of an otherwise uninflamed diverticulum would be sufficient to cause erosion of an underlying vessel with resultant haemorrhage, yet insufficient to cause any other symptoms or evidence of diverticulitis. This is the probable explanation of most cases of bleeding from 'diverticulosis'.

The role of local trauma is difficult to assess. Many of the patients with bleeding from diverticula of the colon whom I have seen have complained of a period of constipation immediately preceding the haemorrhage and this may possibly be of some significance.

A very unusual case of massive colonic bleeding 

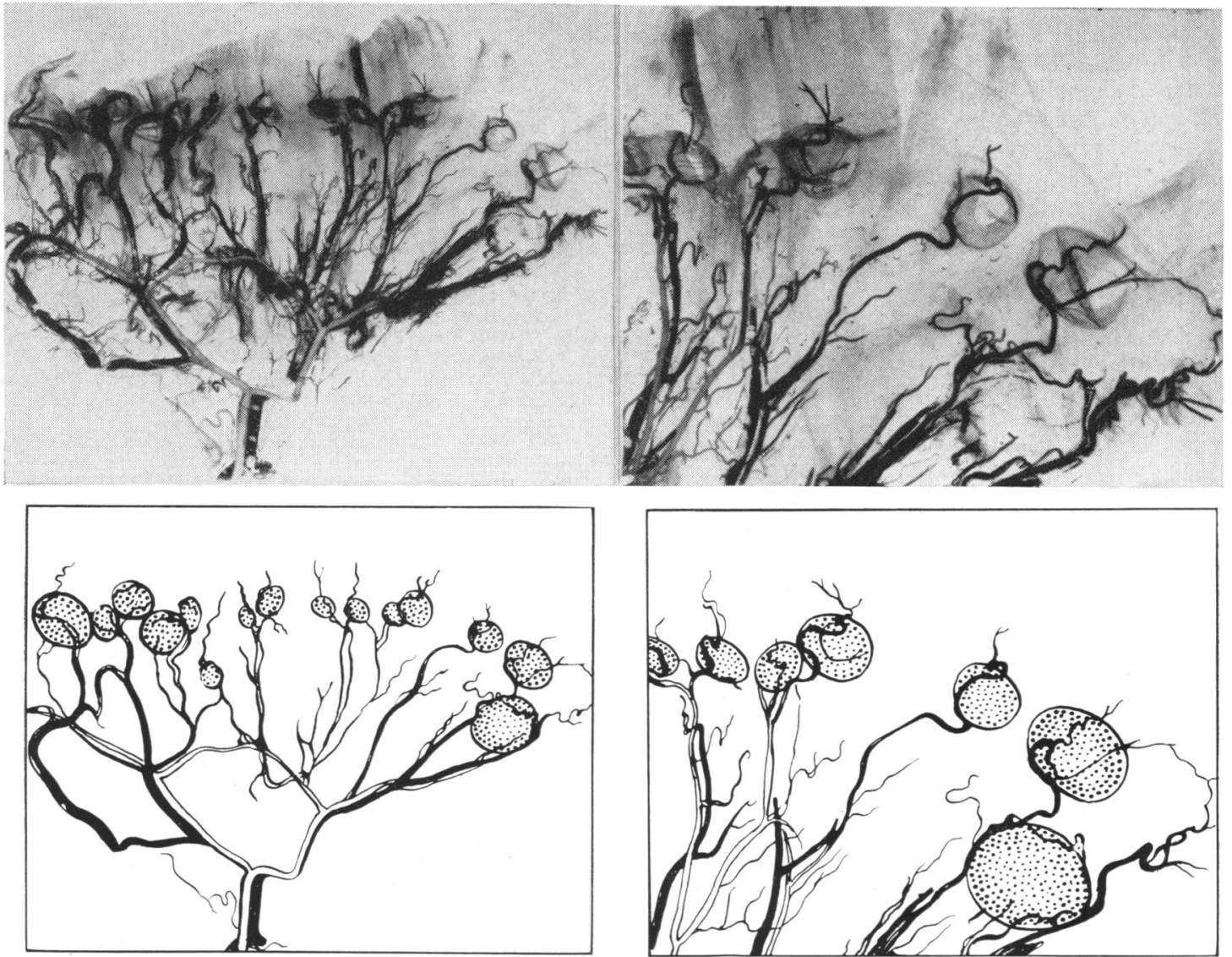

FIG. 1. Specimens of colonic wall prepared by clearing after injection of arteries and veins with red and blue latex. The diagrams indicate the location of diverticula on the photograph (Ann. Surg., 1955, 141, 5).

secondary to diverticulitis has been reported by Hickey (1954). A mass of diverticulitis in the sigmoid colon had become adherent to the antero-lateral abdominal wall; perforation of the diverticulum resulted in a local abscess which eroded the inferior epigastric artery.

\section{DIAGNOSIS}

Unless blood is seen emerging from the mouth of a diverticulum on sigmoidoscopy or the affected colon is resected and examined, the diagnosis has to be made by excluding other causes of bleeding per rectum, particularly polypi and carcinoma of the colon and local conditions in the rectum. This can usually be done in cases of severe or massive haemorrhage, but the minor bleeds are much more difficult to assess, and, although the diagnosis can often be made in these cases, laparotomy has to be resorted to more frequently for this type of bleeding.
Bleeding from carcinoma of the colon is almost invariably slight and usually continuous. Though profuse haemorrhage may occur as an early or presenting symptom of carcinoma, this type of bleeding is decidedly rare in this condition. A sudden profuse haemorrhage occurring in a middle-aged or older person is almost pathognomonic of diverticular disease of the colon.

The sigmoidoscope enables direct inspection of the bowel up to the lower sigmoid region, but above this level reliance has to be placed on barium studies, unless laparotomy is performed. The limitations of opaque enema techniques, particularly in severe diverticulitis, must be borne in mind in assessing the case.

\section{MANAGEMENT}

Conservative treatment, with blood transfusion in the more serious haemorrhages, will arrest the 
bleeding in the majority of cases of bleeding from diverticula of the colon. Haemorrhage may, however, recur. In a small proportion of cases the bleeding will not be arrested by conservative measures and death will result unless surgery is undertaken. A defunctioning colostomy is indicated in these circumstances. A right transverse colostomy is usually the most satisfactory procedure as it allows freedom of access to the left colon for subsequent resection. The colostomy may be permanent, or subsequently the affected colon may be resected with closure of the colostomy. Resection is, of course, essential if carcinoma cannot be excluded with certainty. It may also be wise to prevent recurrent haemorrhage. It is important to realize that haemorrhage may recur in massive form after an unimportant earlier bleed.

Emergency resections during active bleeding should be avoided whenever possible in view of the high mortality they carry.

A careful and prolonged follow-up is essential in all cases.

I gratefully acknowledge the cooperation of the physicians and surgeons of the Central Middlesex Hospital, London, Broadgreen Hospital, Liverpool, and the Royal Southern Hospital, Liverpool, who have allowed me to review their cases, and the assistance I have received from the Radiological and Pathological Departments of the same hospitals.

Figure 1 is reproduced from Annals of Surgery by kind permission of Professor Rudolf J. Noer and Messrs. J. B. Lippincott Company.

\section{REFERENCES}

Bacon, H. E., and Sherman, L. F. (1950). Management of diverticulitis of the sigmoid colon. Amer. J. Surg., 80, 3-10.

Fraenkel, G. J. (1954). Rectal bleeding and diverticulitis. Brit. J. Surg., 41, 643-645.

Germain, A., Le Gallou, M., and Morvan, A. (1938). Forme hémorraguique pure de diverticulose colique à prépondérance droite. Bull. Soc. méd. Hop. Paris, 54, 1430-1434.

Hickey, R. C. (1954). Massive colonic bleeding secondary to diverticulitis. Gastroenterology, 26, 754-757.

Keith, L. M. Jr., and Rini, J. M. (1957). The significance of diverticula of the colon in massive melena. Arch. Surg., 74, 571-573.

Klidjian, A. (1946). Jejunal divertic llosis complicated by haemorrhage. Brit. med. J., 1, 683-684.

Koch, J. (1903). Ueber einfach entzündliche Stricturen des Dickdarms. Langenbecks Arch. klin. Chir., 70, 876-896.

Kozoll, D. D., McMahon, J. A., and Kiely, J. P. (1950). Massive gastrointestinal haemorrhage due to jejunal diverticula. $J$. Amer. med. Ass., 142, 1258-1262.

Lloyd-Davies, O. V. (1953). Diverticulitis. Proc. roy. Soc. Med., 46, 407-416.

Mobley, J. E., Dockerty, M. B., and Waugh, J. M. (1957). Bleeding in colonic diverticulitis. Amer. J. Surg., 94, 44-51.

Morton, J. J., Jr. (1946). Diverticulitis of the colon. Ann. Surg., 124, $725-745$.

Noer, R. J. (1955). Haemorrhage as a complication of diverticulitis. Ibid., 141, 674-685.

Rushford, A. J. (1956). The significance of bleeding as a symptom in diverticulitis. Proc. roy. Soc. Med., 49, 577-579.

Telling, W. H. M. (1908). Acquired diverticula of the sigmoid flexure, considered especially in relation to secondary pathological processes and their clinical symptoms. Lancet, 1, 843-850.

-, and Grüner, O. C. (1917). Acquired diverticula, diverticulitis, and peridiverticulitis of the large intestine. Brit. J. Surg., 4, 468-530.

Waterson, A. P. (1952). Jejunal diverticulosis with haemorrhage. Lancet, 2, 1053-1054.

Young, E. L., and Young, E. L., III. (1944). Diverticulitis of the colon. A review of the literature and an analysis of ninety-one cases. New Engl. J. Med., 230, 33-38.

Young, J. M., and Howorth, M. B. Jr. (1954). Massive haemorrhage in diverticulosis: possible explanation of cause with presentation of case requiring surgical treatment. Ann. Surg., 140, 128-131. 